\title{
BURNOUT Y APOYO SOCIAL EN PERSONAL DEL SERVICIO DE PSIQUIATRÍA DE UN HOSPITAL PÚBLICO
}

\author{
BURNOUT AND SOCIAL SUPPORT STAFF OF A PUBLIC \\ HOSPITAL PSYCHIATRY
}

\author{
Cecilia Avendaño* \\ Patricia Bustos ${ }^{*}$ \\ PAULA EsPINOZA ${ }^{* *}$ \\ Felipe GarCía ${ }^{* * * *}$ \\ TAMARA PIERART ${ }^{* * * *}$
}

\begin{abstract}
RESUMEN
Este artículo presenta resultados de un estudio descriptivo-correlacional que tuvo como objetivos identificar la presencia de burnout y establecer su relación con el apoyo social en el trabajo en personal de un servicio de psiquiatría de un hospital público. Los participantes constituyen el 71\% del personal profesional y de apoyo del servicio. Los resultados muestran que la mayoría de los participantes presenta estrés laboral crónico o burnout, siendo particularmente alta la presencia de agotamiento emocional. Destaca una mayor despersonalización entre quienes realizan turnos y atienden pacientes judicializados, y en los subgrupos de enfermeras, técnicos paramédicos y auxiliares de servicio. Estos resultados señalan la presencia de un grupo ocupacional en situación de vulnerabilidad expresada en un riesgo importante de deterioro de su salud física y mental y con consecuencias laborales negativas sobre la calidad del servicio, la propensión al abandono de la institución, la satisfacción laboral y la tendencia al ausentismo. Por último, la influencia del apoyo social es notable en la disminución de los niveles de agotamiento emocional, despersonalización y baja realización personal, sobre todo cuando proviene de los superiores. El reforzamiento de la capacidad de entregar apoyo de supervisores y jefes mediante un programa de entrenamiento de bajo costo podría tener un efecto altamente positivo en este tipo de servicio.
\end{abstract}

Palabras clave: Burnout, apoyo social, salud mental, personal de salud.

\begin{abstract}
Results of a descriptive-correlational study that it had as objectives to identify the presence of burnout and to establish its relation with the social support in work in personnel of a service of psychiatry of a public hospital, are presented in this article. Participants constitute $71 \%$ of the professional personnel and support of the service. Results show that the majority of the participants displays chronic labor stress or burnout, being particularly high the presence of emotional exhaustion. It emphasizes a greater depersonalization between those who work with patients derived by the judicial system or work on shift, and in the sub-groups of nurses, paramedical and auxiliary technicians on watch. These results indicate the presence of an occupational group in situation of vulnerability expressed in an important risk of deterioration of their physical and mental health and with negative labor consequences on the quality of the service, the propensity to the abandonment of the institution, the labor
\end{abstract}

\footnotetext{
*Dra. en Psicología, profesora del Departamento de Psicología, Facultad de Ciencias Sociales, Universidad de Concepción, Chile. Dirección: ceavendano@udec.cl

**Asistente social, estudiante del Magíster de Psicología, mención Psicología de la Salud, Departamento de Psicología, Facultad de Ciencias Sociales, Universidad de Concepción, Chile. Email: patriciaybt@hotmail.com

***Enfermera, estudiante del Magíster de Psicología, mención Psicología de la Salud, Departamento de Psicología, Facultad de Ciencias Sociales, Universidad de Concepción, Chile. Email: paulyta78@gmail.com

****Psicólogo, Docente de la Universidad San Sebastián y Las Américas, Magíster (c) en Psicología, mención Psicología de la Salud, Departamento de Psicología, Facultad de Ciencias Sociales, Universidad de Concepción, Chile. Email: fgarciam@ udec.cl

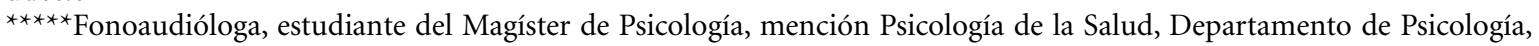
Facultad de Ciencias Sociales, Universidad de Concepción. Chile. Email: tpierart@gmail.com
} 
satisfaction and the tendency to the absenteeism. Finally, the influence of the social support is remarkable in the diminution of the levels of emotional exhaustion, depersonalization and low personal accomplishment, mainly when it comes from the superiors. The reinforcing of the capacity to give support of supervisors and heads by means of a program of training of low cost, could have a highly positive effect in this type of ward.

Key words: Burnout, social support, mental health, health personnel.

Fecha recepción: 17/11/09 Fecha aceptación: 09/06/09

\section{INTRODUCCIÓN}

El burnout es una respuesta al estrés laboral crónico propio de profesiones de servicios caracterizadas por una atención intensa y prolongada a personas en situación de necesidad o dependencia $(1,2)$. El modelo más difundido fue derivado empíricamente de investigaciones realizadas por Maslasch y Jackson, quienes plantearon que era un síndrome compuesto por tres dimensiones: agotamiento emocional, despersonalización y falta de realización personal (3). Agotamiento emocional, se refiere a una pérdida progresiva de la energía vital, mayor irritabilidad y quejas constantes por la cantidad de trabajo, experimentada como estar agotado debido al contacto frecuente y mantenido con personas a las que hay que atender; despersonalización, al desarrollo de actitudes y respuestas distantes y cínicas hacia las personas a quienes se prestan los servicios, y baja realización personal en el trabajo, a la presencia de un autoconcepto negativo y un creciente distanciamiento de actividades familiares y sociales, que afecta el desempeño y la relación con las personas que se atiende (3). El agotamiento emocional representaría la dimensión de tensión básica del síndrome de burnout, en tanto que la despersonalización expresaría el contexto interpersonal en donde las personas desempeñan su trabajo y la baja realización personal en el trabajo representaría una autoevaluación respecto del propio desempeño. Leiter señaló que el burnout puede también ser considerado como una crisis de la autoeficacia (4).

Para su estudio, Maslach y Jackson $(1,2)$ desarrollaron la escala MBI (Maslach Burnout
Inventory) que, hasta la fecha, es el instrumento más utilizado a nivel mundial $(5,6)$.

La perspectiva actual de conceptualización del burnout es psicosocial y define el burnout como un proceso en que intervienen elementos cognitivo-aptitudinales (baja realización personal en el trabajo), emocionales (agotamiento emocional) y actitudinales (despersonalización) (7), y que se diferencia de una perspectiva clínica que lo considera un estado mental (8). Esta distinción conceptual es relevante si se considera el carácter crónico del burnout, es decir, una respuesta prolongada a estresores laborales crónicos (5).

\section{Burnout en personal de salud}

El burnout es muy frecuente en el personal sanitario, entre otros, en psicólogos, psiquiatras, enfermeras, terapeutas ocupacionales, terapeutas familiares y consejeros matrimoniales (9-19). También el personal no sanitario que trabaja en servicios de salud (científicos, obreros de mantenimiento, administrativos y conductores) está expuesto al burnout (20, 21).

Para identificar las variables que actúan como facilitadoras, inhibidoras o que constituyen desencadenantes del burnout en personal sanitario se han investigado diversas variables personales y laborales. Entre las variables personales facilitadoras del burnout, las más estudiadas han sido el género y la edad. Al respecto, algunas investigaciones han encontrado que entre los profesionales sanitarios, las mujeres son el grupo más vulnerable (22), mientras que otras indican que los hombres (19), o bien que no existen diferencias significativas por género (23). 
También los resultados de algunos estudios no encuentran una relación significativa con la edad de los profesionales (23), y en otros el grupo más vulnerable estaría entre los 30 y 39 años de edad (19), mientras un tercer grupo de estudios da cuenta de un mayor nivel de agotamiento emocional y baja realización personal sobre los 44 años (22). En términos generales, los resultados parecen sugerir que la edad no influye directamente en la aparición del burnout, pero que podría relacionarse con una mayor sensibilización en los primeros años de carrera profesional, cuando se produce una transición de las expectativas idealistas hacia la práctica cotidiana y se aprende que las recompensas personales, profesionales y económicas no son ni las prometidas ni las esperadas (22). Sin embargo, en Chile, Melita, Cruz y Merino (24) encontraron más altos niveles de agotamiento emocional en el personal de enfermería de mayor edad.

Entre las variables laborales desencadenantes del burnout, algunas investigaciones establecen una relación directa en los dos primeros años y sobre los diez años de carrera profesional, en cambio otras informan una relación inversa que se explicaría porque aquellos con más años de trabajo tendrían menos burnout y continuarían ejerciendo su profesión, mientras que los que experimentan más burnout la abandonan (25).

Más evidencia favorable se ha observado en la relación entre más horas de trabajo, más pacientes que atender y burnout. En España, por ejemplo, en atención primaria se ha encontrado un nivel de burnout más alto entre quienes tienen un mayor número de enfermos a cargo, permanecen más tiempo interactuando con ellos y tienen un horario más prolongado (22). Y en Chile, Ordenes (26) encontró una relación significativa entre horas trabajadas y ocurrencia de síntomas en trabajadores sanitarios de un hospital (médicos, enfermeras, becados de medicina, kinesiólogos, paramédicos y otros), con un $50 \%$ de casos entre aquellos que trabajaban 44 horas o menos y un $80 \%$ en los que traba- jaban sobre 60 horas, en tanto que Vega et al. (19) observaron un mayor nivel de burnout en psiquiatras de un hospital psiquiátrico que realizaban 21 o más horas semanales de psicoterapia. A su vez, Maslach y Leiter (27) informaron resultados que señalan que los índices de agotamiento emocional son proporcionales al tiempo ocupado en cuidados directos a los enfermos. En personal de enfermería de centros asistenciales chilenos de la Región del Bío Bío que realizaban sobre turno, Melita et al. (24) encontró niveles intermedios de burnout.

En cuanto al esquema de trabajo, Martínez y López (28) han observado diferencias en enfermeras con y sin turnos tanto en despersonalización como en baja realización personal. A su vez, Del Campo, Fernández-Repeto, Martínez y Rojas (29) encontraron que enfermeras con turnos rotatorios puntuaban más alto en baja realización personal, mientras que Albaladejo et al. (30) hallaron puntuaciones más altas en agotamiento emocional, despersonalización y baja realización personal en enfermeras que trabajaban en turnos de tarde o rotatorio. Sin embargo, en estudios con personal de enfermería también se ha encontrado evidencia diferente a la mencionada según el tipo de turno laboral $(23,31,32)$.

En suma, las investigaciones sobre burnout en trabajadores de la salud no son concluyentes respecto del rol facilitador o inhibidor de variables sociodemográficas como el género y la edad, o de factores laborales que podrían actuar como desencadenantes tales como los años de profesión y el esquema de trabajo en turnos, y tampoco se han encontrado relaciones concluyentes entre las variables mencionadas y el nivel de burnout en sus dimensiones específicas (33). Así, el estudio de estas variables continúa siendo importante tanto porque su papel facilitador, inhibidor o desencadenante del burnout como su relación con el impacto del burnout sobre las personas no está todavía suficientemente esclarecido $(7,34)$, dificultando la identificación de los grupos más vulnerables. 


\section{Apoyo social y Burnout}

El apoyo social es considerado uno de los recursos más importantes para manejar y enfrentar estresores cotidianos, eventos vitales o tensiones crónicas, una especie de fondo al cual recurrir en caso de necesidad (35). Su ausencia es en sí misma una fuente de estrés o un factor que contribuye a deteriorar la salud y el bienestar $(36,37)$, y tiene relaciones significativas con la depresión (38).

Es posible distinguir dos niveles de análisis del apoyo social: el estructural y el funcional (39). El estructural está centrado en la dimensión más objetiva de las relaciones que constituyen la red social del individuo, y el funcional en la evaluación subjetiva del apoyo social, especialmente, en las conductas de apoyo -percepción y recepción de apoyo- y en las funciones implicadas en esas conductas. Las redes sociales proporcionan el "marco estructural" y los recursos necesarios para que el apoyo social se haga accesible al individuo desde un "marco funcional" (40). Los cuatro ejes que el conjunto de investigaciones ha permitido identificar como más importantes son los siguientes: (a) para la gente es importante la ayuda percibida como disponible, tanto como la ayuda percibida como efectivamente recibida; (b) el apoyo social puede producirse en tres ámbitos diferentes: la comunidad, las redes sociales y las relaciones íntimas; (c) la ayuda puede ser principalmente de dos tipos: instrumental (como medio para conseguir otros objetivos) o expresiva (como fin en sí misma); y (d) desde el punto de vista del bienestar y la salud, tanto el apoyo cotidiano como el que se recibe en situaciones de crisis son importantes (41).

En relación al burnout, diversas investigaciones muestran que un apoyo inadecuado, o que no se brinda, facilita su aparición y el desarrollo de enfermedades (42), y que el apoyo social está negativamente asociado con burnout $(7,41-45)$. También se ha encontrado que los compañeros de trabajo y el jefe inmediato constituyen fuentes de apoyo importantes (46), asociadas con una menor incidencia de burnout (33). En enfermeras chilenas existe alguna evidencia de que la relación con los pares está relacionada con el agotamiento emocional y la baja realización personal ${ }^{1}$.

En general, la literatura sobre apoyo social en el trabajo permite afirmar que éste ha sido el recurso más investigado en su relación al burnout y que la evidencia en relación con la importancia de la falta de apoyo es fuertemente consistente, especialmente en la falta de apoyo que brindan los jefes o supervisores más que en la falta de apoyo de los compañeros de trabajo $(5,47)$.

Frente a los antecedentes anteriores, la presente investigación intenta contribuir a identificar cuál es el rol de algunas variables sociodemográficas y laborales previamente señaladas como importantes en la aparición del síndrome y establecer qué relación hay entre burnout y apoyo social en trabajadores de la salud chilenos que trabajan en servicios psiquiátricos, sector muy poco estudiado en nuestro país.

Los objetivos del estudio son: (a) establecer la presencia de burnout según edad, género, sistema de turnos, tipo de turnos, antigüedad en el sector salud y nivel ocupacional en el personal del Servicio de Psiquiatría del Hospital Guillermo Grant; (b) establecer las relaciones entre niveles de burnout según edad, género, sistema de turnos, tipo de turnos, antigüedad en el sector salud y nivel ocupacional; y (c) establecer las relaciones entre niveles de burnout por dimensión y percepción de apoyo social recibido.

${ }^{1}$ Saavedra C. Carga laboral percibida y burnout en profesionales de Enfermería que trabajan en hospital y Centros de Salud Familiar de las comunas de Talcahuano y Hualpén, año 2005. [Tesis para optar al grado de Magíster en Enfermería]. Concepción, Chile: Departamento de Enfermería, Facultad de Medicina, Universidad de Concepción; 2006. 
Las hipótesis a comprobar fueron las siguientes:

1. Las mujeres presentan un mayor agotamiento emocional que los hombres.

2. Los hombres presentan una mayor despersonalización que las mujeres.

3. Los participantes ubicados en rangos de edad extremos presentan puntuaciones más altas en alguno de los tres factores del burnout.

4. Los participantes con una antigüedad laboral inferior a 2 años y superior a 22 años presentan puntuaciones más altas en los tres factores del burnout.

5. Los participantes que trabajan en el sector público del servicio presentan puntuaciones más altas en alguna de las dimensiones del burnout que aquellos que trabajan en el sector privado del mismo servicio.

6. Los participantes que trabajan en un sistema de turnos presentan puntuaciones más altas en alguna de las dimensiones del burnout que aquellos que no realizan turnos.

7. Los participantes que trabajan en sistemas de turnos rotatorios presentan puntuaciones más altas en alguno de las dimensiones del burnout que aquellos que trabajan en sistemas de turnos fijos.

8. La percepción de apoyo social recibido total, de los superiores y compañeros de trabajo, se relaciona con mayor agotamiento emocional, mayor despersonalización y menor realización personal.

\section{MATERIAL Y MÉTODO}

El estudio tuvo un diseño transversal de tipo descriptivo y correlacional. Los participantes fueron 98 personas $(71 \%)$ de un total de 137 que trabajan a tiempo completo o parcial en el Servicio de Psiquiatría señalado, esto es, todo el personal de planta y contrata de este servicio. Por sexo, son 55 mujeres (56\%) y 43 hombres (44\%), con una edad media de
35 años $(\mathrm{DS}=9,4)$ en un rango comprendido entre los 22 años y 62 años. Entre los participantes hubo16 médicos, 7 becados de medicina, 10 enfermeras, 5 psicólogos, 33 técnicos paramédicos 11 administrativos, 10 auxiliares de servicio y otros 6 profesionales. Su antigüedad laboral promedio es de 7 años $(\mathrm{DS}=7,6)$, en un rango que va entre menos de un año y 35 años. Un 56\% trabaja en turnos, y de ellos un $80 \%$ lo hace en turnos rotatorios y un $20 \%$ en sistema de turnos de fin de semana. En sistema de turnos un 34\% son técnicos paramédicos, $11 \%$ auxiliares de servicio, $7 \%$ enfermeras, $2 \%$ médicos, $y$ $2 \%$ otros.

\section{INSTRUMENTOS Y VARIABLES}

Los siguientes son las variables del estudio y los instrumentos utilizados para evaluarlas:

1. Burnout: se evaluó mediante el Maslach Burnout Inventory (MBI) para profesionales sanitarios (2). La escala utilizada posee originalmente 22 ítemes, pero, después de ser validada para esta investigación mediante un Análisis de Componentes Principales con método Varimax, que confirmó la estructura de tres factores de la versión original, quedó compuesta por 19 ítemes. Los tres factores explican un $58,3 \%$ de la varianza total. La consistencia interna fue establecida con el coeficiente Alfa de Cronbach, siendo de 0,90 para la escala total, 0,87 para agotamiento emocional, 0,78 para despersonalización y 0,75 para baja realización personal, valores aceptables y concordantes con los de otros estudios (7). Las respuestas se presentan en una escala tipo Likert de 6 alternativas $(1=$ nunca, $2=$ una vez al año o menos, $3=$ una vez al mes o menos, $4=$ una vez por semana, $5=$ algunas veces por semana y $6=$ todos los días). Siguiendo las recomendaciones de Maslach y Jackson (2), por encima del percentil 75 los participantes fueron clasificados en la categoría "con burnout" para la puntuación 
total o "alto nivel" en una dimensión específica; sobre el percentil 50 como "proclives al burnout" para la puntuación total o "mediano nivel" en una dimensión específica, y por debajo del percentil 25 "sin burnout" para la puntuación total o "bajo nivel" en una dimensión específica.

2. Percepción de apoyo social recibido en el trabajo: se utilizó una escala compuesta por 6 ítemes relativos a percepción de apoyo social recibido en el trabajo del Cuestionario de Evaluación de Riesgos Psicosociales en el Trabajo ISTAS21 (CoPsoQ), que es la adaptación para el Estado español del Cuestionario Psicosocial de Copenhague (CoPsoQ) (48). Un ítem se refiere a la percepción de apoyo recibido de los compañeros de trabajo, otro al apoyo recibido de los superiores, dos a sentirse incluido, y dos son de apoyo recibido general. Las respuestas se presentan en una escala tipo Likert de 6 alternativas $(0=$ nunca, $1=$ sólo alguna vez, $2=$ algunas veces, $3=$ muchas veces, y $4=$ siempre). Para este estudio, la consistencia interna de la escala calculada con el coeficiente Alfa de Cronbach es 0.83 , lo que se considera aceptable. Las puntuaciones en apoyo social total o según la fuente (compañeros o superiores) fueron clasificadas de la siguiente manera: con puntuaciones sobre el percentil 75 los participantes fueron clasificadas en la categoría con "alto nivel de apoyo", sobre el percentil 50 con "mediano nivel de apoyo", y por debajo del percentil 25 con "bajo nivel de apoyo".

3. Las variables sociodemográficas consideradas fueron edad y sexo, y las variables laborales ocupación, antigüedad en el servicio, esquema de trabajo (con sistema de turnos o sin turnos), el tipo de turnos realizados (rotatorio o de fin de semana), y sector en que trabaja en el servicio de salud (público/ privado $)^{2}$.

\section{PROCEDIMIENTO}

El estudio fue autorizado por el director del hospital, quien también asumió la responsabilidad de motivar la colaboración del personal del Servicio de Psiquiatría. Los participantes fueron invitados a participar mediante una carta de consentimiento que explicaba los objetivos del estudio y garantizaba anonimato, confidencialidad y voluntariedad de la participación. Además, los investigadores estuvieron disponibles para responder dudas. El dilema ético que significaba la identificación de participantes en niveles de burnout con riesgo para su salud fue enfrentado ofreciendo los resultados individuales para orientar a una atención de salud específica y entregando los resultados globales a la autoridad del servicio para que tomara las medidas de apoyo correspondientes. El cuestionario fue aplicado en dos formas: en reuniones clínicas del equipo de salud y en una reunión de técnicos paramédicos, y en forma individual a aquellos que no participaron de esas reuniones. Los participantes podían devolver personalmente el cuestionario a los investigadores, o dejarlo en secretaría.

$\mathrm{Al}$ iniciarse la aplicación del cuestionario, diez personas estaban con licencia médica y siete no fueron ubicadas porque sus turnos no coincidieron con la distribución de los cuestionarios. Finalmente, fueron distribuidos 120 cuestionarios y 102 devueltos; cuatro fueron eliminados del análisis por contener omisiones o errores. Las razones entregadas para no participar fueron desconfianza en el anonimato del estudio, desinterés por conocer el índice de burnout del personal o falta de tiempo para contestar.

Para el análisis de los datos descriptivos se utilizó medidas de tendencia central y dispersión, t de Student y Anova; en el análisis

${ }^{2}$ En este caso, la diferencia fundamental entre trabajar en el sector público o privado del servicio es que en el público, además de los pacientes habituales, se atiende a pacientes judiciales, es decir personas que han cometido delitos y que son derivados por los tribunales de justicia al sistema de salud público. 
de las relaciones fue utilizada la correlación de Pearson, y en las asociaciones de los datos por niveles tau b de Kendall; por último, para una relación causal fue empleado un análisis de regresión lineal simple. El procesamiento de los datos fue realizado con los programas SPSS 15.0 y Excel.

\section{RESULTADOS}

\section{Resultados en las dimensiones del bur- nout}

La puntuación total en el MBI ubica al 30\% de los participantes en la categoría sin bur- nout, al $49 \%$ en la categoría de proclividad al burnout y al $20 \%$ en la categoría con burnout (ver Gráfico 1). En la Tabla 1 puede observarse que la puntuación media más alta obtenida por los participantes es en agotamiento emocional, seguida por baja realización y despersonalización. En agotamiento emocional casi un $70 \%$ de los participantes está ubicado en un nivel de proclividad al burnout o con burnout, y en las dimensiones despersonalización y baja realización un $66 \%$ está en los mismos niveles. Un porcentaje más alto de participantes presenta alto nivel de baja realización $(26 \%)$ y despersonalización $(22 \%)$ que de agotamiento emocional $(20 \%)$.

Tabla 1. Resultados descriptivos en las dimensiones del burnout

\begin{tabular}{|l|c|c|}
\hline Dimensión & Media & DE \\
\hline Agotamiento emocional & 3,8 & 1,2 \\
Despersonalización & 2,7 & 1,2 \\
Realización personal en el trabajo & 2,1 & 0,9 \\
\hline
\end{tabular}

En el análisis de diferencias realizado para las variables sociodemográficas y laborales consideradas en el estudio se observó que la puntuación media en agotamiento emocional es levemente mayor para los hombres $(3,9)$ que para las mujeres $(3,7)$; en despersonalización los hombres obtuvieron una media de 2,8 y las mujeres $2,5 \mathrm{y}$, por último, en baja realización personal la media fue 2 para los hombres y 2,2 para las mujeres. Sin embargo, estas diferencias no son estadísticamente significativas, lo que refuta las hipótesis 1 y 2. Por edad existen diferencias significativas en la dimensión de baja realización personal $(\mathrm{F}=2,169, \mathrm{p}=, 004)$, pero, un ANOVA con los datos de edad reagrupados permitió observar que en edades extremas las diferencias no son significativas, lo que refuta la hipótesis 3 . Por su parte, en antigüedad laboral no se observan diferencias significativas (agotamiento emocional, $\mathrm{F}=, 968, \mathrm{p}=, 411$; despersonalización, $\mathrm{F}=, 484, \mathrm{p}=, 694$; realización personal, $\mathrm{F}=1,150, \mathrm{p}=, 333$ ), lo que refuta la hipótesis 4 . En cambio, se observa una diferencia significativa en despersonalización según el sector en que el personal realiza sus actividades $(t=2,147, p=043)$, siendo mayor la media de quienes trabajan en el sector público del servicio $(2,9)$ versus la media de aquellos que trabajan en el sector privado $(2,1)$, lo que confirma la hipótesis 5.

En la variable turnos se observa una diferencia significativa $(t=, 718, p=, 008)$ en la puntuación media de despersonalización entre quienes realizan turno $(2,9)$ versus quienes no los realizan $(2,3)$. También se produce esta diferencia en despersonalización entre los subgrupos ocupacionales que trabajan en turnos ( $\mathrm{F}=4,309, \mathrm{p}=, 041)$, esto es, enfermeras, técnicos paramédicos y auxiliares de servicio, 
respecto de los subgrupos que no trabajan en sistema de turnos, es decir, médicos, asistentes sociales, psicólogos, becados de medicina y administrativos, lo que confirma hipótesis 6 . Sin embargo, en ninguna dimensión se producen diferencias entre quienes realizan turnos rotatorios o de fin de semana (agotamiento, emocional, $\mathrm{t}=-, 041, \mathrm{p}=12,3$; despersonalización, $\mathrm{t}=, 368, \mathrm{p}=15,3$; baja realización, $\mathrm{t}=, 347$, $\mathrm{p}=13,8)$, lo que refuta la hipótesis 7 .

\section{Resultados en apoyo social percibido en el trabajo}

En la escala de apoyo social la media del grupo estudiado en la puntuación total fue 2,8 $(\mathrm{DE}=1)$. El Gráfico 1 permite apreciar que un $71,5 \%$ de los participantes están ubicados en los niveles de mediano y alto apoyo social total percibido. Además, la puntuación media en percepción de apoyo recibido de compañeros $(2,9)$ es más alta que la media de percepción de apoyo recibido de superiores $(2,5)$.

Para las variables sexo, edad, antigüedad en el servicio, nivel ocupacional, trabajar en sistema de turnos y tipo de turno no hay diferencias significativas en las percepciones de apoyo social recibido total y de apoyo recibido de superiores. En cambio, la media en apoyo recibido de los compañeros es más alta entre quienes realizan turnos rotatorios que entre quienes realizan turnos de fin de semana $(3,1$ y 2,3, respectivamente) siendo significativa esa diferencia $(t=2,2, p=, 049)$.

Por otra parte, en el análisis por dimensiones del burnout pueden apreciarse diferencias para percepción de apoyo social recibido total (despersonalización, $\mathrm{F}=2,080$, $\mathrm{p}=, 019$; baja realización personal, $\mathrm{F}=2,124$, $\mathrm{p}=, 016$; agotamiento emocional, $\mathrm{F}=2,033$, $\mathrm{p}=, 022)$, y también para apoyo social de los superiores (despersonalización, $\mathrm{F}=5,109$, $\mathrm{p}=, 001$; baja realización personal, $\mathrm{F}=2,572$, $\mathrm{p}=, 043$; agotamiento emocional, $\mathrm{F}=3,212$, $\mathrm{p}=, 016)$. Ello indica que la percepción de apoyo recibido en general y de los compañeros de trabajo y superiores tiene influencia en puntuar diferentemente en las dimensiones del burnout.

Gráfico 1. Resumen niveles en las dimensiones de burnout y en apoyo social

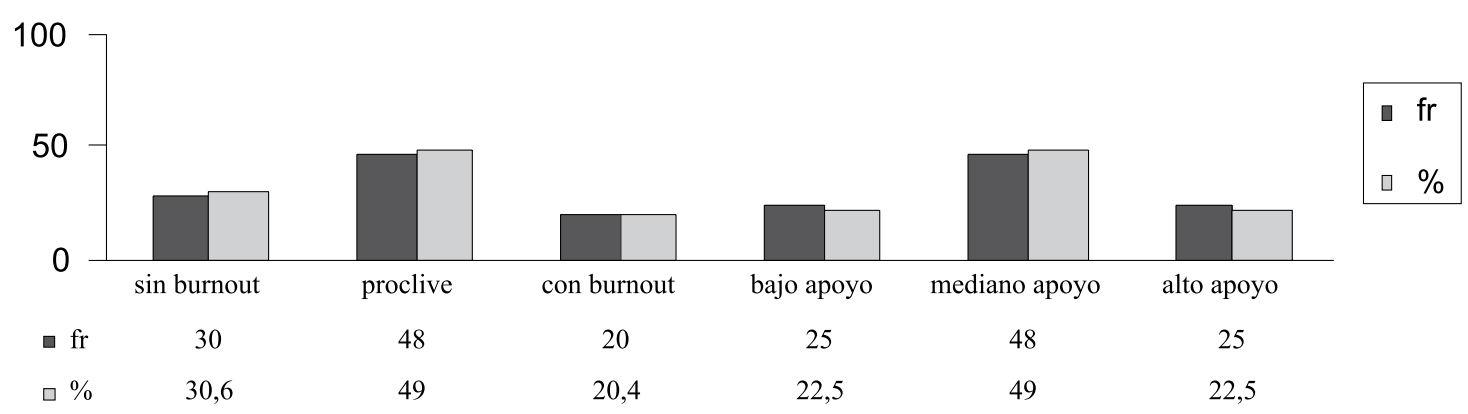

3. Resultados en la relación apoyo social percibido en el trabajo y burnout

Del análisis correlacional realizado se concluyó que hay relaciones inversas y moderadas estadísticamente significativas entre todos los tipos de percepción de apoyo social recibido (total, de los superiores y de los compañeros de trabajo) y las tres escalas del burnout (ver Tabla 2), siendo levemente más fuerte la relación entre el apoyo recibido de los superiores con despersonalización. Estos datos permiten concluir que una mayor percepción de apoyo social recibido en el trabajo, ya sea de apoyo total como de los 
compañeros o de los superiores, influye en una menor presencia de agotamiento emocional, despersonalización y baja realización personal, en particular, que a más percepción de apoyo recibido de los superiores habría menos despersonalización.

Tabla 2. Correlaciones entre apoyo social y dimensiones del burnout

\begin{tabular}{|c|c|c|c|c|}
\hline & & apoyo total & $\begin{array}{c}\text { apoyo } \\
\text { superiores }\end{array}$ & $\begin{array}{c}\text { apoyo } \\
\text { compañeros }\end{array}$ \\
\hline \multirow[t]{3}{*}{ despersonalización } & $\begin{array}{l}\text { Correlación de } \\
\text { Pearson }\end{array}$ &,$- 316^{* *}$ &,$- 335^{\star \star}$ &,$- 244^{\star}$ \\
\hline & Sig. (bilateral) & 0,002 & 0,001 & 0,0015 \\
\hline & $\mathrm{N}$ & 98 & 98 & 98 \\
\hline \multirow[t]{3}{*}{ baja realización } & $\begin{array}{l}\text { Correlación de } \\
\text { Pearson }\end{array}$ &,$- 280^{\star *}$ &,$- 225^{\star \star}$ &,$- 269^{\star}$ \\
\hline & Sig. (bilateral) & 0,005 & 0,026 & 0,007 \\
\hline & $\mathrm{N}$ & 98 & 98 & 98 \\
\hline \multirow[t]{3}{*}{ agotamiento } & $\begin{array}{l}\text { Correlación de } \\
\text { Pearson }\end{array}$ &,$- 267^{\star *}$ &,$- 235^{\star \star}$ &,$- 253^{\star}$ \\
\hline & Sig. (bilateral) & 0,008 & 0,02 & 0,012 \\
\hline & $\mathrm{N}$ & 98 & 98 & 98 \\
\hline
\end{tabular}

* La correlación es significante al nivel 0,05 (bilateral).

** La correlación es significativa al nivel 0,01 (bilateral).

Un análisis de regresión realizado para la dimensión despersonalización y apoyo recibido del superior como variable explicativa confirma la existencia de una relación causal entre ambas variables. La despersonalización es explicada en un $11,3 \%$ por el apoyo social recibido de los superiores $\left(\mathrm{R}^{2}=.112,-.335, \mathrm{t}=\right.$ $3,487$, sig. $=, 001)$, indicando que el trabajador experimentará menos despersonalización a mayor apoyo social percibido del superior. Todos estos resultados confirman la hipótesis número 8. La varianza no explicada señala la necesidad de estudiar otras variables que den cuenta de la despersonalización.

\section{DISCUSIÓN}

Los resultados obtenidos muestran que un
69\% de los participantes se ubican en un nivel de proclividad al burnout (49\%) o con burnout $(20,4 \%)$. Estas cifras son semejantes a las que han sido encontradas en países europeos y más bajas que en los Estados Unidos, cuando se ha utilizado el MBI como instrumento de medición (5). Para el caso chileno la evidencia es escasa todavía y no existen criterios normativos establecidos en el nivel nacional que permitan comparar estos resultados.

Por dimensiones, la media en agotamiento emocional es más alta $(3,8)$ que la despersonalización $(2,1)$ y la baja realización en el trabajo $(2,7)$, cifras que dan cuenta de un alto nivel de agotamiento emocional y niveles medianos de despersonalización y baja realización en el trabajo. En diversos estudios en que se ha intentando establecer el orden de aparición de los síntomas que integran el burnout, el 
agotamiento emocional aparece como precursor del desarrollo del proceso de burnout. Leiter (50) y Maslach y Leiter (27) señalan que en primer lugar surgían sentimientos de agotamiento emocional que llevaban a la despersonalización, la que a su vez produciría baja realización personal en el trabajo. Posteriormente, otros hallazgos llevaron a Leiter (51) a proponer que el agotamiento precede a la despersonalización, y que la baja realización surgiría en forma paralela al agotamiento emocional, y sería una consecuencia directa de la falta de apoyo social y de oportunidades para desarrollarse en el trabajo. Este último modelo y otras variaciones propuestas más tarde son el foco de muchas investigaciones y todavía no son concluyentes respecto de su nivel de generalización $(51,52)$, siendo de gran relevancia para la comprensión del desarrollo del burnout y la intervención preventiva. No obstante, las características del diseño de este estudio sólo permiten sugerir que el riesgo de burnout en este grupo es importante para un porcentaje notable de los participantes, pero no permiten aventurar conclusiones definitivas sobre el desarrollo del burnout en esta muestra. Para profundizar en el conocimiento del desarrollo del burnout, o del burnout como proceso, se requiere realizar otras investigaciones con diseño longitudinal.

Por otra parte, en despersonalización se observó diferencias entre quienes realizan turnos versus quienes no los realizan, entre quienes atienden pacientes judicializados versus quienes no los atienden. Asimismo, en esta dimensión del burnout aparecen diferencias entre los subgrupos ocupacionales que trabajan en turnos (enfermeras, técnicos paramédicos y auxiliares de servicio, y dos médicos) versus los subgrupos que no trabajan en este sistema (la mayoría de los médicos participantes, psicólogos, becados de medicina y administrativos). Los datos muestran que existe mayor despersonalización entre quienes realizan turnos y atienden pacientes judicializados, y en los subgrupos de enfermeras, técnicos paramédicos y auxiliares de servicio.
Este resultado es particularmente relevante en un contexto en que la atención de pacientes judicializados ha sido amplia y públicamente expuesta como un problema grave por los trabajadores de los servicios de psiquiatría del sector público de salud. Podría hipotetizarse que la despersonalización constituye una estrategia de administración del estrés generado por la interacción con pacientes que implican un manejo especialmente difícil para el personal que mantiene un mayor contacto directo, y muy demandante, situación que podría estar siendo evaluada como una exigencia que sobrepasa los mecanismos habituales de afrontamiento de las tensiones (crónicas, en este caso) y que se traduce en distanciamiento de esos pacientes y, sobre todo, en centrar la atención en sus características negativas (53). En investigaciones realizadas en otros países con funcionarios de hospitales psiquiátricos que atienden usuarios del ámbito penitenciario se han encontrado relaciones significativas con despersonalización, atribuidas a las características de los usuarios, y también entre esta dimensión y el manejo de la relación, atribuidas a las dificultades y conflictos que el personal sanitario debe enfrentar cotidianamente con ellos (34).

Además, una relación significativa entre apoyo social y burnout observada indica que en presencia de una mayor percepción de apoyo social recibido en el trabajo, ya sea de compañeros o de superiores, es menor el agotamiento emocional, la despersonalización y la baja realización personal en el trabajo. Este resultado ha sido visto en forma consistente en un gran número de investigaciones realizadas en diversos países y coincide con abundante evidencia que señala que en el plano de las relaciones interpersonales en el trabajo, la falta de apoyo aumenta los sentimientos de burnout en las profesiones de la salud, o que su presencia disminuye la influencia del burnout (7). Asimismo, una relación causal observada en este estudio entre percepción de apoyo social recibido de los superiores y despersonalización ha sido informada anteriormente 
en profesores (54) y auxiliares de enfermería (55). Por otra parte, que la percepción de apoyo social recibido de los compañeros sea mayor que el apoyo recibido de superiores, sumado a que la relación entre apoyo de los superiores con despersonalización es más fuerte que con agotamiento emocional y baja realización en el trabajo, sugiere que en este grupo existe el riesgo que el apoyo social recibido de los superiores, tal como está siendo percibido, no lo proteja suficientemente de desarrollar actitudes negativas hacia los usuarios de sus servicios y, por el contrario, podría estar facilitando el surgimiento de estas actitudes. La mayor importancia del apoyo percibido de los superiores en el trabajo, respecto del apoyo de los compañeros, está bien establecida en la literatura especializada $(5,47)$.

A pesar de sus limitaciones de diseño, este estudio proporciona información útil para la elaboración de un programa de administración del estrés crónico en servicios de psiquiatría del sector público. A futuro, es necesario continuar investigando en personal de servicios de psiquiatría las variables personales y laborales que predisponen, precipitan y perpetúan el burnout en el largo plazo (56, 57), replicando este estudio en otros servicios de psiquiatría para poder realizar comparaciones e incluyendo variables que no fueron incluidas, tales como el rol moderador de las estrategias de afrontamiento del estrés, tipo de paciente, interacción paciente-personal, entre otras. Asimismo, el apoyo social en el trabajo debe ser investigado en otras dimensiones de la percepción, por ejemplo, la percepción de apoyo disponible de diversas fuentes laborales.

En conclusión, la mayoría del personal profesional y de apoyo del Servicio de Psiquiatría estudiado evidencia estrés laboral crónico o burnout, siendo particularmente alta la presencia de agotamiento emocional. Destaca también la mayor despersonalización entre quienes realizan turnos y atienden pacientes judicializados, y en los subgrupos de enfermeras, técnicos paramédicos y auxiliares de servicio. Estos resultados señalan la presencia de un grupo ocupacional en situación de vulnerabilidad expresada en un riesgo importante de deterioro de su salud física y mental y con consecuencias laborales negativas sobre la calidad del servicio, la propensión al abandono de la institución, la satisfacción laboral y la tendencia al ausentismo. Por último, la influencia del apoyo social es notable en la disminución de los niveles de agotamiento emocional, despersonalización y baja realización personal, sobre todo cuando proviene de los superiores. El reforzamiento de la capacidad de entregar apoyo de supervisores y jefes mediante un programa de entrenamiento de bajo costo podría tener un efecto altamente positivo en este tipo de servicio, contribuyendo a aminorar los efectos estresantes del trabajo, mejorando los sentimientos de baja realización, disminuyendo el agotamiento emocional y moderando la despersonalización, al menos parcialmente.

\section{REFERENCIAS}

1. Maslach C, Jackson S. The measurement of experienced burnout. J Organ Behav 1981; 2: 99-113.

2. Maslach C, Jackson S. MB1: The Maslach Burnout Inventory: Manual Research. Palo Alto, CA: Consulting Psychologists Press; 1986.

3. Maslach C, Jackson S, Leiter M. Maslach Burnout Inventory Manual. 3rd ed. Palo Alto, CA: Consulting Psychological Press; 1996.

4. Leiter M. Burnout as a crisis in self-efficacy. Conceptual and practical implications. Work and Stress 1992; 6 (2): 107-116.

5. Maslach C, Schaufeli W, Leiter M. Job burnout. Annu Rev Psychol 2001; 52: 397-422.

6. Ortega C, López F. El burnout o síndrome de estar quemado en los profesionales sanitarios: Revisión y perspectivas. Int J Clin 
Health Psychol 2004; 4(1):137-160.

7. Gil-Monte P, Peiró J. Desgaste psíquico en el trabajo: El síndrome de quemarse. Madrid: Síntesis; 1997. Pp. 13-15.

8. Schaufeli WB, Enzmann D. The burnout companion to study and practice: A critical analysis. London: Taylor \& Francis; 1998. Pp. 1-17.

9. Benavides Pereira AM, Moreno-Jiménez B, Garrosa Hernández E, González Gutiérrez JL. La evaluación específica del síndrome de burnout en psicólogos: El inventario de burnout de psicólogos. Clínica y Salud 2002; 13(3): 257-283.

10. Corrigan P, Colmes P, Luchins D, Buican B. Staff burnout in a psychiatric hospital: A cross-lagged panel design. J Organ Behav 1994; 15: 65-74.

11. González J, Moreno R, Peñacoba C, Alcocer N, Alonso L, del Barco P. Burnout in occupational therapy: An analysis focused on the level of individual and organizational consequences. Psychology in Spain 2004; 8(1): 98-105.

12. Korkeila J, Töyry S, Kumpulainen K, Toivola J, Räsänen K, Kalimo R. Burnout and self-perceived health among finnish psychiatrists and child psychiatrist: A national survey. Scand J Publ Health 2003; 31(2): 85-91.

13. Mehta R. Burnout in clinical psychologists in the UK: An examination of its nature, extent and correlates. [Doctoral dissertation]. Lancaster, UK: Institute for Health Research, Lancaster University; 2006.

14. Rafii F, Oskouie F, Nikravesh M. Factors involved in nurses' responses to burnout: A grounded theory study. BMC Nursing [online] 2004; 3(6). [cited 2008 marzo 3]; Disponible en: http://www.biomedcentral. com/1472-6955/3/6

15. Hernández R. Estrés y burnout en profesionales de la salud de los niveles primario y secundario de atención. Rev Cubana Salud Pública 2003; 29(2):103-110.

16. Rosenberg T, Pace M. Burnout among mental health professionals: Special considerations for the marriage and family therapist. J Marital Fam Ther 2006; 32(1):87-98.

17. Thomsen S, Soares J, Nolan P, Dallender J, Arnetz, B. Feelings of professional fulfilment and exhaustion in mental health personnel: The importance of organizational and individual factors. Psychother Psychosom 1999; 68:157164.

18. Torrado V, Calvo C, Fernández L. Occupational stress and state of health among clinical psychologists and psychiatrists. Psychology in Spain 1997; 1(1):63-71.

19. Vega S, Yáñez J, González M, Quezada M. (2004). El desgaste personal de los psiquiatras: Síndrome de burnout (resultados preliminares). Santiago, Chile: Centro de Psicología Aplicada, Universidad de Chile; 2004.

20. Anderson WJR, Cooper CL, Willmott M. Sources of stress in the national health service: A comparison of seven occupational groups. Work and Stress 1996; 10(1):88-95.

21. Pardo J, López F, Moriña M, Pérez M, Freire P, Fernández R. ¿Estamos quemados en la atención primaria? Med. Fam. (And) 2002; 4:245-250.

22. Atance J. Aspectos epidemiológicos del síndrome de burnout en personal sanitario. Rev. Esp. Salud Pública 1997; 71(3):293-303.

23. Pando M, Bermúdez D, Aranda C, Pérez J, Flores E, Arellano G. Prevalencia del estrés y el burnout en los trabajadores de la salud en Cd. Guayana, Venezuela. Psicología y Salud 2003; 13(1):47-52.

24. Melita A, Cruz M, Merino JM. Burnout en profesionales de Enfermería que trabajan en centros asistenciales de la Octava Región, Chile. Cienc. Enferm. 2008, 14(2):75-85.

25. Naisberg JL, Fenning S. Personality characteristics and propeness to Burnout: 
A study among psychiatrits. Stress Med 1991; 7(4):201-205.

26. Ordenes N. Prevalencia de burnout en trabajadores del Hospital Roberto del Río. Rev. Chil. Pediatr. 2004; 75(5):449-454.

27. Maslach C, Leiter M. The impact of interpersonal environment on burnout and organizational commitment. J Organ Behav 1988; 9:297-308.

28. Martínez C, López G. Características del síndrome de burnout en un grupo de enfermeras mexicanas. Arch Med Fam 2005; 7(1): 6-9.

29. Del Campo M, Fernández-Repeto E, Martínez J, Rojas A. El síndrome de quemarse por el trabajo (burnout) en los profesionales de Enfermería de las unidades de diálisis de la provincia de Cádiz. XXIV Congreso Nacional de la Sociedad Española de Enfermería Nefrológica 1999. Valencia, España [en línea] 1999. [cited 2007 Jul 27]; Disponible en: http://www. seden.org

30. Albaladejo R, Villanueva R, Ortega P, Astasio $\mathrm{P}$, Calle M, Domínguez V. Síndrome de burnout en el personal de enfermería de un hospital de Madrid. Rev. Esp. Salud Pública 2004; 78(4): 505-516.

31. Marrero M. Burnout en enfermeros que laboran en los servicios de neonatología de Ciudad Habana. Revista Cubana de Salud y Trabajo 2003; 4(1-2):33-38.

32. Jofré V, Valenzuela S. Burnout en personal de Enfermería de la unidad de cuidados intensivos pediátricos. Aquichan 2005; 5(5):56-63.

33. Moreno B, Peñacoba C. Estrés asistencial en los servicios de salud. En: Simon MA (ed.). Psicología de la Salud. Madrid: Pirámide; 1999 . Pp. 739-764.

34. García JM, Herrero S. Variables sociodemográficas y síndrome de burnout en una muestra multiocupacional del Hospital Psiquiátrico Penitenciario de Sevilla. Apunt. Psicol. 2008; 26(3):459-477.

35. Thoits P. Stress, coping, and social support processes: Where are we? What next? J
Health Soc Behav (Extra Issue) 1995; 53-79.

36. Scharzer R, Leppin A. Social support and health: A theoretical and empirical overview. J Soc Pers Relat 1991; 8:99127.

37. Payne R, Jones G. Measurement and methodological issues in social support. En: Kasl SV \& Cooper CL (Eds.). Research methods in stress and Health Psychology. Chichester: Wiley \& Sons; 1987. Pp. 167205.

38. Páez, D. Suceso vital, soporte social, recursos e identidades sociales como factores explicativos de la ansiedad y de la depresión. En: Páez D (ed.). Salud mental y factores psicosociales. Madrid: Fundamentos. 1986. Pp. 83-127.

39. Abril VJ. Apoyo social y salud. Valencia: Promolibro; 1997. Pp. 8-11.

40. Lin N, Dean A, Ensel W. Social Support Scales: A Methodological Note. Schizophr Bull 1981; 7:73-90.

41. Gracia E, Herrero J, Musitu G. El apoyo social. Barcelona: PPU; 1995. Pp. 22-26.

42. Aranda C, Pando M, Pérez M. Apoyo social y síndrome de quemarse en el trabajo o burnout: Una revisión. Psicología y Salud 2004; 14(1):78-97.

43. Gil-Monte P. El síndrome de quemarse por el trabajo (Síndrome de Burnout) en profesionales de Enfermería. Inter Ação Psy [en línea] 2003; 1:19-33 [cited 2007 Jul 27]. Disponible en: http://www.cepis. org.pe/bvsacd/cd49/artigo3.pdf

44. Baruch-Feldman C, Brondolo E, BenDayan D, Schwartz J. Sources of social support and burnout, job satisfaction, and productivity. J Occup Health Psychol 2002; 7(1):84-93.

45. Brown C, O'Brien KM. Understanding stress and burnout in shelter workers. Prof Psychol Res Pract 1998; 29:383-385.

46. Greenglass ER, Fiksenbaum L, Burke RJ. The relationship between social support and burnout over time in teachers. J Soc BehavPers 1994; 9:219-230. 
47. Bakker AB, Demerouti E, Verbeke W. Using the Job Demands-Resources Model to predict burnout and performance. JHRM 2004; 43:83-104.

48. Instituto Sindical de Trabajo, Ambiente y Salud [ISTAS]. Método Istas 21 (CoPsoQ). Manual para la evaluación de riesgos psicosociales en el trabajo. España: Paralelo Edición; 2004.

49. Leiter M. Conceptual implications of two models of burnout. Group Organ Stud 1989; 14(1):15-22.

50. Leiter M. Burnout as a development process: Considerations of models. En Schaufeli W, Maslach C, Marek T (Eds.). Professional burnout: Recent development in theory and research. Washington DC: Hemisphere; 1993. p. 115-129.

51. Gil-Monte P, Peiró J, Valcárcel P. A model of burnout process development: An alternative from appraisal models of stress. COeG 1998; 4(1):165-179.

52. Leiter M, Gascón S, Martínez-Jarreta B. A two process model of Burnout: Their relevance to spanish and canadian nurses. Psychol Spain 2008; 12(1):37-45.

53. Fothergill A, Edwards D, Burnard P. Stress, burnout, coping and stress management in psychiatrists: Findings from a systematic review. Int J Soc Psychiatry 2004; 50:54-65.

54. Rusell D, Altmaier E, Velzen D. Job-related stress, social support, and burnout among classroom teachers. J Appl Psychol 1987; 72(2):269-274.

55. Vinaccia S, Alvaran L. (2004). El síndrome del burnout en una muestra de auxiliares de Enfermería: Un estudio exploratorio. Ter psico (Impr.) 2004; 22(1):9-16

56. Kumar S, Hutcher S, Huggard P. Burnout in psychiatrists: An etiological model. Int J Psychiatr Med 2005; 35(4):405-416.

57. Kumar S, Fisher J, Robinson E, Hatcher S, Bhagat R. Burnout and job satisfaction in New Zealand psychiatrists: A national study. Int J Psychiatr Med 2007; 53(4):306316. 